\title{
Cimento aluminoso e seus efeitos em concretos refratários magnesianos espinelizados in situ
}

\section{(Calcium aluminate cement and its effects on in-situ spinel containing magnesia refractory castables)}

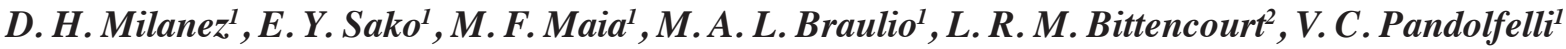 \\ ${ }^{I}$ Grupo de Engenharia de Microestrutura de Materiais - GEMM \\ Departamento de Engenharia de Materiais, Universidade Federal de S. Carlos \\ Rod. Washington Luiz, km 235, C.P. 676, S. Carlos, SP 13565-905 \\ ${ }^{2}$ Magnesita S.A., Centro de Pesquisas e Desenvolvimento - CPqD, Praça Louis Ensch 240, Contagem, MG \\ vicpando@power.ufscar.br
}

\begin{abstract}
Resumo
O uso de concretos refratários alumina-magnésia ligados por cimento aluminoso (CAC) apresenta vantagens decorrentes da presença de espinélio e de $\mathrm{CA}_{6}\left(\mathrm{CaO} .6 \mathrm{Al}_{2} \mathrm{O}_{3}\right)$, ambas formadas in-situ e acompanhadas de expansão. Estas fases possuem alta resistência a escórias básicas e ao choque térmico, propriedades estas imprescindíveis para aplicação em panelas siderúrgicas. Estudos anteriores mostraram que o teor de CAC utilizado em concretos alumina-magnésia influencia a expansão do material, principalmente devido à formação de $\mathrm{CA}_{6}$. Este trabalho visa estudar a influência do teor de cimento no sistema magnésia-alumina, utilizando-se a mesma matriz de um concreto alumina-magnésia tradicional. Os resultados indicaram que o CAC influencia na estabilidade volumétrica do sistema MgO-espinélio: quanto menor o teor de CAC, menor a retração das amostras. Isso refletiu na sinterização dos concretos e, assim, nas propriedades mecânicas após queima em temperaturas elevadas.

Palavras-chave: concretos magnesianos, espinélio, cimento de aluminato de cálcio.
\end{abstract}

\begin{abstract}
Calcium aluminate cement (CAC) bonded alumina-magnesia refractory castables present greatadvantages for steel ladle applications as a result of in-situ expansive formation of spinel and $C A_{6}$, which leads to high basic slag and thermal shock resistance. The $C A C$ content in those castables strongly influences its expansive behavior mainly due to $C_{6}$ formation. In the present work, the effects of the CAC content in magnesia-alumina castables were analyzed. The results showed that calcium aluminate cement affects the volumetric stability of $\mathrm{MgO}$-spinel system: the lower the CAC content, the lower the shrinkage. These effects on the sintering and in the mechanical properties after sintering at high temperatures are also presented and discussed.
\end{abstract}

Keywords: magnesia castable, spinel, calcium aluminate cement.

\section{INTRODUÇÃO}

Os agregados constituem a grande maioria dos concretos refratários. A literatura indica que a substituição dos agregados aluminosos por magnesianos, em concretos espinelizados in situ contendo a mesma matriz, resulta em fases distintas para os dois sistemas e afeta o comportamento expansivo em altas temperaturas [1]. Adicionalmente, as fases resultantes previstas em análises envolvendo somente a matriz dos concretos são distintas das encontradas experimentalmente. Por exemplo, no sistema alumina-magnésia verifica-se a formação de $\mathrm{CA}_{6}$ e espinélio causando a expansão dos concretos em altas temperaturas, ao passo que no sistema magnésia-alumina as fases formadas são espinélio e belita $\left(\mathrm{C}_{2} \mathrm{~S}\right)$ resultando em retração em temperaturas similares [1]. Este mesmo silicato também é encontrado em estudos de reações em tijolos básicos [2].

Quanto aos ligantes para concretos refratários, o uso do cimento de aluminato de cálcio é o mais comum em sistemas contendo $\mathrm{Al}_{2} \mathrm{O}_{3}$ e $\mathrm{MgO}[3,4]$. Autores deste trabalho mostraram a influência do teor de cimento na hidratação do $\mathrm{MgO}$ e na expansão causada pela formação de espinélio e CA ${ }_{6}$ [5]. Adicionalmente, as propriedades termomecânicas foram analisadas, mostrando a importante função dos grãos aciculares de $\mathrm{CA}_{6}$ no ancoramento das trincas geradas $[6,7]$. Neste sentido, diversos trabalhos apresentam o $\mathrm{CA}_{6}$ como agente tenacificador, melhorando as propriedades como choque térmico, fluência e resistência mecânica a quente. Com elevada refratariedade e morfologia acicular, o $\mathrm{CA}_{6}$ atua também como elemento de ligação entre a matriz e os agregados [6-9]. Assim como nos aluminosos, o teor de CAC 
utilizado deve também atuar no comportamento expansivo e a resistência mecânica de concretos magnesianos. Deste modo, o objetivo deste trabalho foi avaliar o efeito da variação do teor de CAC no comportamento expansivo e na formação de fases em concretos ricos em $\mathrm{MgO}$ espinelizados in situ. Em complemento, um software de simulação termodinâmica foi utilizado para ajudar na previsão das fases formadas em temperaturas elevadas e no entendimento do comportamento expansivo destes concretos magnesianos.

\section{MATERIAIS E MÉTODOS}

Utilizando-se o modelo de empacotamento de Alfred, dois concretos foram formulados com coeficiente de empacotamento $\mathrm{q}=0,26$, sendo o primeiro aluminoso, contendo agregados de alumina tabular $(\mathrm{d} \leq 6 \mathrm{~mm}$, Almatis, Alemanha), e o outro magnesiano, com agregados eletrofundidos (98\%-p de magnésia, $\mathrm{d} \leq 4,75 \mathrm{~mm}, \mathrm{CaO} /$ $\mathrm{SiO}_{2}=3,5$, Magnesita, Brasil). A matriz destes concretos foi projetada do mesmo modo para os dois materiais, sendo composta por alumina reativa (CL370C, Almatis, Alemanha), sínter de $\mathrm{MgO}$ (95\%-p de magnésia, $\mathrm{CaO} /$ $\mathrm{SiO}_{2}=0,36, \mathrm{~d} \leq 45 \mu \mathrm{m}$, Magnesita, Brasil), microssílica (971U, Elkem Materials, Norway) e cimento de aluminato de cálcio (Secar 71, Kerneos, França). A Tabela I apresenta as formulações utilizadas.

Tabela I - Formulações utilizadas.

[Table I - Compositions analyzed.]

\begin{tabular}{ccc}
\hline Matéria Prima & $\begin{array}{c}\text { Alumina- } \\
\text { magnésia }\end{array}$ & $\begin{array}{c}\text { Magnésia- } \\
\text { alumina }\end{array}$ \\
\hline $\mathrm{AT}(6,0 \mathrm{~mm}>\mathrm{d}>0,2 \mathrm{~mm})$ & 62 & - \\
$\mathrm{AT}(\mathrm{d}<0,2 \mathrm{~mm})$ & 18 & 8 \\
$\mathrm{ME}(\mathrm{d}<4,75 \mathrm{~mm})$ & - & 72 \\
$\mathrm{CL} 370 \mathrm{C}$ & 7 & 7 \\
Sinter MgO & 6 & 6 \\
CAC & $6,4,2$ & $6,4,2$ \\
Microssílica & 1 & 1 \\
\hline
\end{tabular}

AT: Alumina Tabular; ME: Magnésia Eletrofundida.

A matriz dos concretos foi projetada para obtenção de 21\%-p de espinélio. Para atender esta finalidade, foi necessário acrescentar 8\%-p de alumina tabular $(\mathrm{d} \leq 200 \mu \mathrm{m})$ no concreto magnesiano, Tabela I. Os teores de 6, 4 e 2\%-p de CAC foram ajustados em ambos os concretos. A dispersão do sistema foi obtida pelo uso de um policarboxilato (Bayer, Alemanha), levando a um consumo de água de 4\%-p para ambos os concretos. Estes materiais foram misturados em um reômetro por cinco minutos, seguindo a metodologia de adição de água em duas etapas, conforme Pileggi e autores $[10,11]$ sugerem.

Um equipamento de refratariedade sobre carga (RUL 421E, Netzsch, Alemanha) foi utilizado para o ensaio de sinterabilidade assistida destes materiais. Corpos cilíndricos de $50 \mathrm{~mm}$ de altura foram preparados segundo a norma DIN 51053, com diâmetro externo de $50 \mathrm{~mm}$ e interno de $12,4 \mathrm{~mm}$. Estas amostras foram moldadas, curadas a $50{ }^{\circ} \mathrm{C}$ em ambiente saturado de água por um dia, secas a $110^{\circ} \mathrm{C}$ por $24 \mathrm{~h}$ e calcinadas a $600{ }^{\circ} \mathrm{C}$ por $5 \mathrm{~h}$. Posteriormente, as amostras foram ensaiadas até $1500{ }^{\circ} \mathrm{C}$ a $3{ }^{\circ} \mathrm{C} / \mathrm{min}$ e $5 \mathrm{~h}$ de patamar. A carga de compressão utilizada foi $0,02 \mathrm{MPa}$.

Para ajudar no entendimento dos resultados de expansão, foi feita análise de microscopia eletrônica de varredura (BSI) e EDS por meio de um equipamento Hitachi S-510.

Para análise das propriedades mecânica e da variação

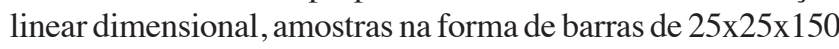
$\mathrm{mm}^{3}$ foram preparadas, curadas $\left(1\right.$ dia a $50{ }^{\circ} \mathrm{C}$, ambiente saturado em água), secas $\left(1\right.$ dia a $\left.110^{\circ} \mathrm{C}\right)$ e pré-queimadas a $600{ }^{\circ} \mathrm{C}$ por $5 \mathrm{~h}$. Os corpos foram, então, sinterizados a $1{ }^{\circ} \mathrm{C} /$ min em três temperaturas por 5 h: 1150,1300 e $1500^{\circ} \mathrm{C}$. O módulo de ruptura destes materiais foi obtido por flexão em três pontos em uma máquina universal de ensaios mecânicos (810, MTS Systems), de acordo com a norma ASTM C13394. Foi também realizado o ensaio de porosidade aparente pelo o método de Arquimedes, norma ASTM C830.

\section{RESULTADOS E DISCUSSÃO}

O uso de ligantes (CAC) levou à avaliação do seu teor sobre a hidratação do $\mathrm{MgO}$, formação de espinélio e estabilidade volumétrica de concretos alumina-magnésia [5]. Com a redução do teor de cimento, houve diminuição do pico de decomposição de brucita observado durante a secagem do concreto e da expansão em temperaturas elevadas, devido à redução na formação de $\mathrm{CA}_{6}$. Em concretos magnesianos, a hidratação do $\mathrm{MgO}$ é inevitável, pois esta matéria-prima é utilizada em quantidades elevadas (80\%-p neste trabalho). Porém, com o controle da dispersão e empacotamento de partículas, aliado ao uso de agregados eletrofundidos que possuem menor área superficial, o teor de água utilizado para moldagem foi baixo. Com tais medidas, não foram identificadas trincas associadas à formação de brucita durante

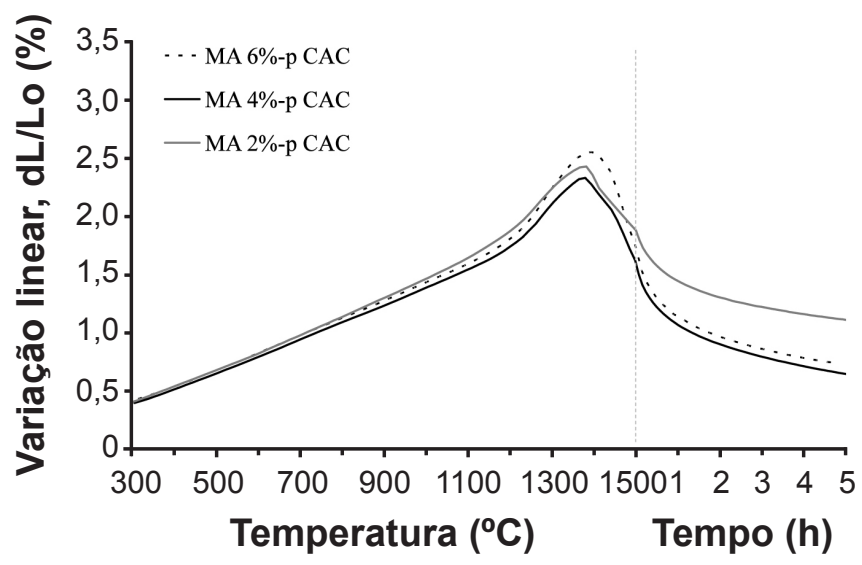

Figura 1: Curvas de expansão de concretos magnesianos espinelizados in situ, contendo teores de CAC distintos.

[Figure 1: Expansion behavior of in situ magnesia spinel castables with different CAC content.] 
as etapas de cura e secagem. O comportamento expansivo dos concretos magnésia-alumina foi avaliado, visando-se sua correlação com as fases que podem ser obtidas em altas temperaturas. A Fig. 1 apresenta as curvas de sinterabilidade assistida destes concretos para diferentes teores de CAC.

Mesmo com a variação no teor de CAC, as curvas apresentaram comportamentos semelhantes: expansão com o aumento de temperatura, mudança na inclinação da curva de expansão com a formação de espinélio $\left(\sim 1300{ }^{\circ} \mathrm{C}\right)$ e posterior retração em temperaturas superiores à $1400^{\circ} \mathrm{C}$. A Tabela II apresenta os valores percentuais de retração a partir do amolecimento $\left(\sim 1400{ }^{\circ} \mathrm{C}\right)$ observado na Fig. 1, deixando mais claro que a diminuição do teor de CAC, implica na

Tabela II - Valores de retração percentual a partir da temperatura de início de amolecimento.

[Table II - Shrinkage percentual values after the softening starting temperature.]

\begin{tabular}{cc}
\hline Composição & \% de retração \\
\hline MA 6\%-p CAC & 1,82 \\
MA 4\%-p CAC & 1,68 \\
MA 2\%-p CAC & 1,32 \\
\hline
\end{tabular}

redução da retração obtida.

Este comportamento não foi observado em concretos alumina-magnésia, uma vez que naquele sistema a variação do teor de CAC levou a diminuição na expansão dos concretos [6].

Para auxiliar na avaliação do comportamento dos concretos ricos em MgO, a Fig. 2 apresenta a derivada das curvas de expansão.

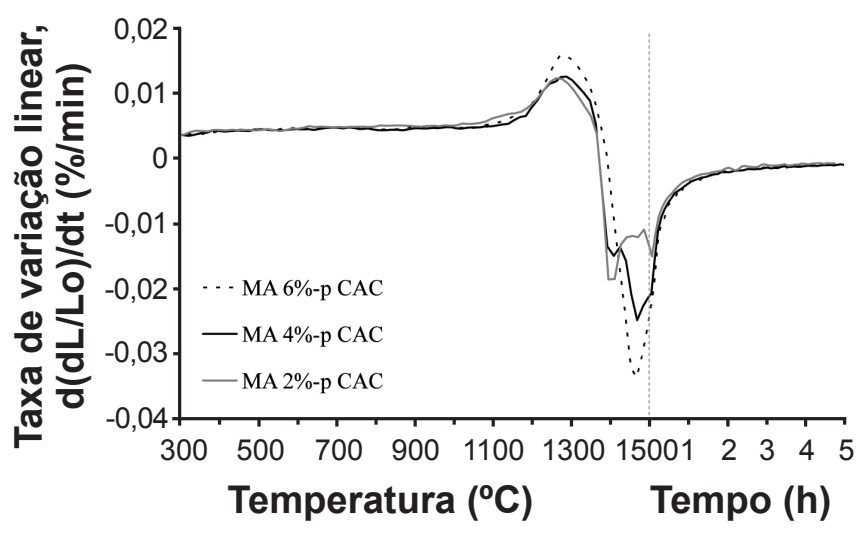

Figura 2: Taxa de variação linear de concretos magnesianos espinelizados in situ, contendo teores de CAC distintos.

[Figure 2: Expansion rate of in situ magnesia spinel castables with different CAC content.]

Aspectos relevantes são apresentados na Fig. 2. O primeiro pico detectado $\left(\sim 1300{ }^{\circ} \mathrm{C}\right)$ indica a formação de espinélio, como discutido em um trabalho anterior [5]. Após o ponto de máximo da curva, a taxa de variação linear foi negativa, atribuído à sinterização e a formação de fase líquida $\left(\mathrm{C}_{12} \mathrm{~A}_{7}\right)$ [1]. Adicionalmente, as amostras apresentaram comportamentos distintos em temperaturas próximas de $1450{ }^{\circ} \mathrm{C}$. A composição contendo 6\%-p de cimento apresentou maior taxa de retração, seguido do concreto com 4 e $2 \%$-p de cimento.

Com a finalidade de se investigar estas diferenças, foram realizadas análises microestruturais dos concretos magnésiaalumina e do alumina-magnésia, contendo 6\%-p de CAC e queimados a $1500{ }^{\circ} \mathrm{C}$. A Fig. 3 ilustra estas microestruturas.

Em várias regiões representativas destas amostras a análise de EDS foi efetuada. Com relação à composição
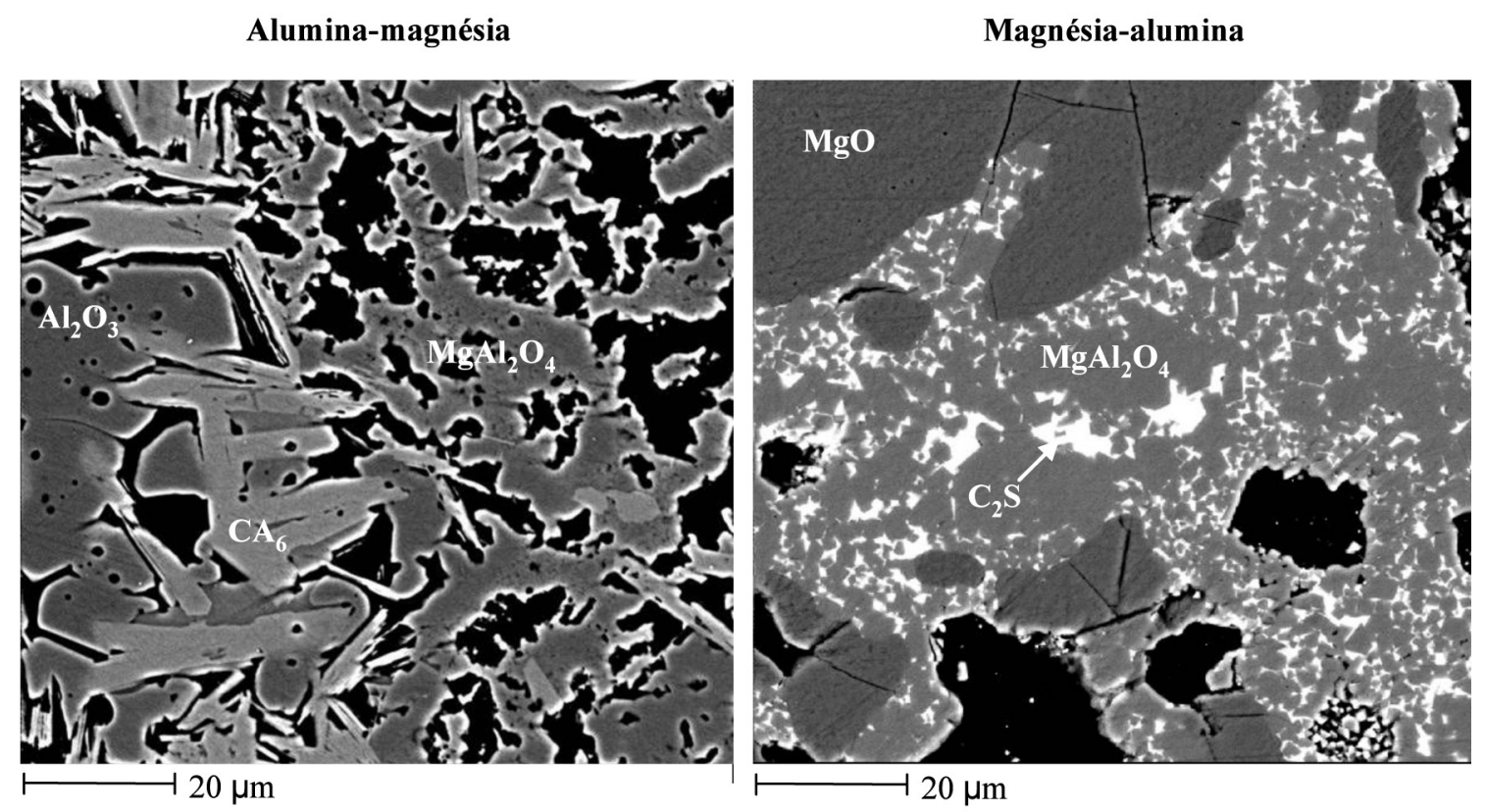

Figura 3: Micrografias do sistema alumina-magnésia e do sistema magnésia-alumina após sinterização a $1500{ }^{\circ} \mathrm{C}$ por $5 \mathrm{~h}$.

[Figure 3: Microstructural features of alumina-magnesia and magnesia-alumina refractory castables after sintering at $1500{ }^{\circ} \mathrm{C}$ for $5 \mathrm{~h}$.] 
Tabela III - Simulações termodinâmicas dos concretos alumina-magnésia e magnésia-alumina. [Table III - Thermodynamic simulations for the alumina-magnesia and magnesia-alumina castables.]

\begin{tabular}{|c|c|c|c|c|c|c|}
\hline \multirow{2}{*}{$\%-\mathrm{p}$} & \multicolumn{3}{|c|}{$\mathrm{AM}$} & \multicolumn{3}{|c|}{ MA } \\
\hline & $6 \%-$ - CAC & $4 \%$-p CAC & $2 \%$-p CAC & $6 \%$-p CAC & $4 \%$-p CAC & $2 \%-\mathrm{p} \mathrm{CAC}$ \\
\hline $\mathrm{MgAl}_{2} \mathrm{O}_{4}$ & 21,0 & 21,0 & 21,0 & 26,7 & 25,6 & 24,5 \\
\hline $\mathrm{CA}_{6}$ & 14,3 & 9,0 & 1,8 & - & - & - \\
\hline $\mathrm{CA}$ & - & - & - & 0,3 & 0,2 & 0,1 \\
\hline $\mathrm{C}_{2} \mathrm{~S}$ & - & - & - & 2,6 & 1,8 & 1,0 \\
\hline $\mathrm{CAS}_{2}$ & 2,2 & 2,3 & 2,3 & - & - & - \\
\hline $\mathrm{C}_{2} \mathrm{AS}$ & 0,4 & - & - & - & - & - \\
\hline $\mathrm{Al}_{2} \mathrm{O}_{3}$ & - & 5,5 & 12,8 & - & - & - \\
\hline $\mathrm{MgO}$ & - & - & - & 0,1 & 0,1 & - \\
\hline
\end{tabular}

magnésia-alumina, verificou-se o $\mathrm{MgO}$ eletrofundido nos agregados e o espinélio $\left(\mathrm{MgAl}_{2} \mathrm{O}_{4}\right)$ e a belita $\left(\mathrm{C}_{2} \mathrm{~S}\right)$ na matriz. Com relação à belita, as características das reações de tijolos magnésia-espinélio em contato com o banho de cimento de fornos rotativos, foram analisadas, observando as mesmas fases obtidas neste estudo [12]. Para a composição aluminamagnésia foi identificada a alumina tabular, espinélio na matriz e $\mathrm{CA}_{6}$ nitidamente reagido ao redor dos agregados aluminosos, como discutido por diversos autores $[6,8,9]$. Após a identificação dessas fases nos dois tipos de concretos, uma simulação termodinâmica (FactSage, Thermfact and GTT-Technologies, Universidade Federal de São Carlos, FAI) foi realizada para os concretos $\mathrm{Al}_{2} \mathrm{O}_{3}-\mathrm{MgO}$ e $\mathrm{MgO}-\mathrm{Al}_{2} \mathrm{O}_{3}$ com teores distintos de $\mathrm{CAC}$, encontrando-se as possíveis fases formadas no equilíbrio a $1500{ }^{\circ} \mathrm{C}$. A Tabela III indica os resultados destas simulações.

Para o sistema alumina-magnésia os valores termodinamicamente previstos de espinélio foram os mesmos para os três teores de CAC. No sistema magnésiaalumina a quantidade de espinélio formada é superior que no sistema alumina-magnésia, pois a alumina do cimento foi utilizada para formação adicional de espinélio. Assim, dois aspectos merecem destaque baseado nestes resultados: com a diminuição do teor de CAC no sistema magnésiaalumina, houve uma redução do teor de alumina para formar espinélio; por outro lado, no sistema alumina-magnésia, o componente limitante é a magnésia, indicando que após seu consumo não há formação adicional de espinélio. Quanto às fases envolvendo o $\mathrm{CaO}$, os teores de $\mathrm{CA}_{6}$ no sistema alumina-magnésia e os de $\mathrm{C}_{2} \mathrm{~S}\left(\mathrm{C}: \mathrm{CaO}\right.$ e $\left.\mathrm{S}: \mathrm{SiO}_{2}\right)$ e CA (A: $\mathrm{Al}_{2} \mathrm{O}_{3}$ ) no magnésia-alumina diminuem com a redução do teor de cimento. No sistema aluminoso, a anortita $\left(\mathrm{CAS}_{2}\right)$ foi prevista e em quantidades iguais para os três teores de CAC, indicando que a sílica afeta consideravelmente o desenvolvimento destas fases. Traços de guelenita $\left(\mathrm{C}_{2} \mathrm{AS}\right)$ também foram obtidos para o concreto aluminoso com 6\%-p CAC.

Correlacionando-se os resultados de sinterabilidade assistida e de simulação termodinâmica, os diferentes picos observados a $1450{ }^{\circ} \mathrm{C}$ nos concretos magnesianos podem ser identificados (Fig. 2). Em decorrência do menor teor de $\mathrm{CaO}$ e considerando-se o caminho de reação das fases associadas ao $\mathrm{CAC}$ de 1300 para $1500{ }^{\circ} \mathrm{C}\left(\mathrm{CA}_{2} \rightarrow \mathrm{C}_{12} \mathrm{~A}_{7} \rightarrow \mathrm{CaO}\right)$ [1], o teor de fase transiente $\mathrm{C}_{12} \mathrm{~A}_{7}$ é menor, diminuindo a quantidade de fase líquida desenvolvida. Com a menor presença de fase líquida, a retração é reduzida. Sendo assim, menor a taxa de retração para o concreto contendo 2\%-p CAC, seguido do concreto com 4\%-p CAC e 6\%-p CAC. Adicionalmente, na curva do concreto magnesiano com 2\%-p CAC (Fig. 2) o pico apresentado em $1450{ }^{\circ} \mathrm{C}$ possui concavidade para baixo, ou seja, indica expansão. Tal comportamento se deve, possivelmente, a formação de espinélio secundário da reação da alumina com os grãos de $\mathrm{MgO}$ eletrofundidos, resultando em um menor valor final de expansão, como pode ser analisado na Fig. 1. Esse mesmo comportamento é observado na curva do concreto com 4\%-p de CAC, porém em menor intensidade devido à maior quantidade de fase líquida encontrada neste concreto. Para o concreto com 6\%-p de CAC, este pico não é observado, pois nesse material o teor de fase líquida é superior. Deste modo, a retração se sobrepõe a expansão por formação de espinélio secundário.

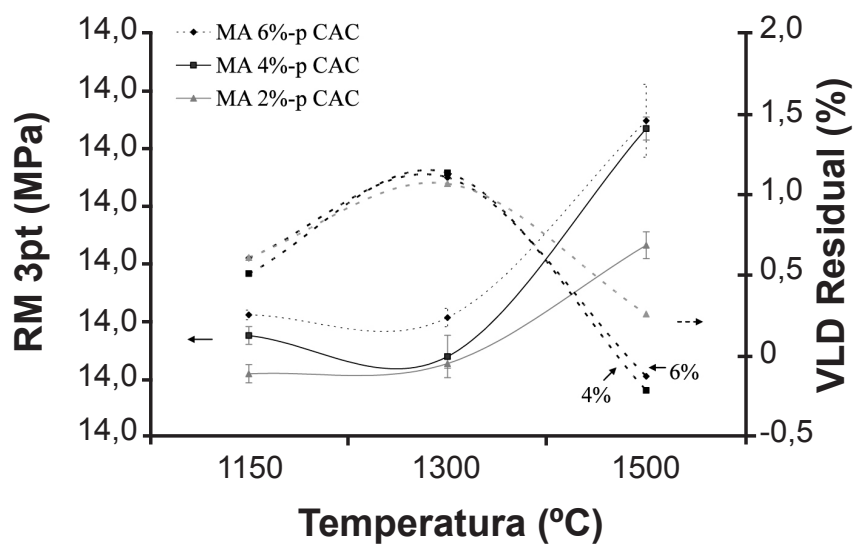

Figura 4: Resistência mecânica e VLD residual dos concretos magnesianos espinelizados in situ com diferentes teores de CAC. [Figure 4: Mechanical behavior and permanent linear expansion of in situ magnesia spinel containing different CAC content.] 


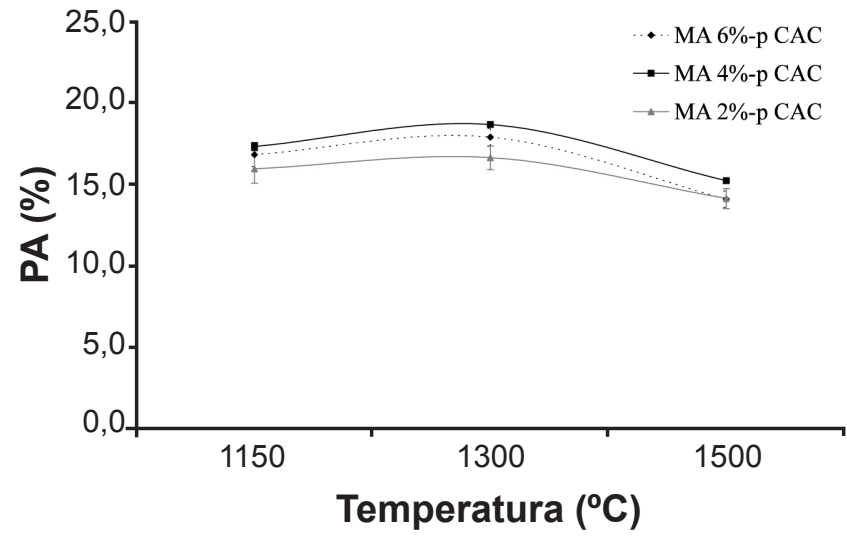

Figura 5: Porosidade aparente em função da temperatura de queima dos concretos magnesianos espinelizados in situ com diferentes teores de CAC.

[Figure 5: Apparent porosity of in situ magnesia spinel containing different CAC content.]

Com o entendimento do comportamento expansivo e das fases formadas, os resultados de resistência mecânica, variação linear dimensional e porosidade aparente após queima dos concretos magnesianos com diferentes teores de cimento são apresentados nas Figs. 4 e 5, após queima a 1150,1300 e $1500{ }^{\circ} \mathrm{C}$.

Em relação à VLD (variação linear dimensional), os resultados indicam valores próximos no intervalo de 1150 e $1300{ }^{\circ} \mathrm{C}$, independentemente da quantidade de cimento, devido à formação de espinélio. Por outro lado, os valores à $1500{ }^{\circ} \mathrm{C}$ seguiram tendências próximas às das curvas de sinterabilidade assistida: o concreto com 2\%-p de CAC apresentou valor residual superior que os concretos com 6 e 4\%-p de CAC. Quanto à resistência mecânica, a redução de CAC resultou em menores módulos de ruptura nas temperaturas de 1150 e $1300{ }^{\circ} \mathrm{C}$. Já a $1500^{\circ} \mathrm{C}$ os valores medidos são superiores aos encontrados nas outras temperaturas para todos os teores de CAC. Assim como ocorreu nas curvas de VLD houve uma aproximação dos resultados de módulo de ruptura dos concretos com 4 e $6 \%$-p de CAC. Tal fato está ligado ao grau semelhante de sinterização destes dois concretos mostrado pela VLD.

Nos resultados de porosidade aparente percebe-se a proximidade dos valores percentuais e as tendências dos concretos magnesianos contendo diferentes teores de CAC. Entre 1150 e $1300{ }^{\circ} \mathrm{C}$ há aumento na porosidade aparente dos concretos devido à espinelização in-situ, e a $1500^{\circ} \mathrm{C}$ verificase redução dos valores em decorrência da sinterização ter prevalecido sobre a expansão.

\section{CONCLUSÕES}

De modo distinto ao observado em concretos aluminosos contendo magnésia, os concretos magnesianos contendo alumina apresentaram retração durante a etapa de sinterização. Assim como no sistema alumina-magnésia, o teor de cimento também afetou a estabilidade volumétrica destes concretos ricos em $\mathrm{MgO}$. Com relação a este aspecto, o aumento no teor de CAC resultou em maior retração, possivelmente devido à maior formação da fase transiente $\mathrm{C}_{12} \mathrm{~A}_{7}$ e da sinterização. Por meio da análise da taxa de variação linear, a menor retração dos sistemas contendo 2 e 4\%-p CAC evidenciou uma expansão adicional em temperaturas próximas a $1450{ }^{\circ} \mathrm{C}$, atribuída à formação de um espinélio secundário devido à reação entre a alumina presente no cimento e os grãos de magnésias eletrofundidas. Este fenômeno destacou mais uma vez a falta de inércia dos agregados refratários, uma vez que o sínter fino de $\mathrm{MgO}$ já havia reagido com a alumina presente no concreto. Tais resultados estão de acordo com as previsões termodinâmicas obtidas, que indicaram nos concretos magnesianos, maior formação de espinélio com o aumento do teor de CAC. Já para a variação do teor de cimento em concretos aluminamagnésia, a simulação destacou a diminuição da formação de $\mathrm{CA}_{6}$, que resulta na menor expansão do material. Porém, o teor de CAC não afetou o teor de anortita neste sistema. Estas variações microestruturais, além de afetar a expansão dos sistemas, também influenciaram o desenvolvimento da resistência mecânica e da porosidade aparente dos concretos analisados. Diferentemente do concreto alumina-magnésia, no qual o aumento de temperatura resulta em expansão excessiva e maior porosidade aparente, acarretando menor resistência mecânica para concretos com maior teor de CAC [6], os concretos magnesianos apresentaram outra tendência. Com o aumento do teor de cimento, maior foi a resistência mecânica e menor porosidade aparente após sinterização a $1500{ }^{\circ} \mathrm{C}$, em decorrência da maior retração.

\section{AGRADECIMENTOS}

Os autores agradecem ao suporte fornecido pela FAPESP, CNPq e Magnesita S.A. para a realização deste trabalho.

\section{REFERÊNCIAS}

[1] M. A. L. Braulio, D. H. Milanez, E. Y. Sako, L. R. M. Bittencourt, V. C. Pandolfelli, Are Refractory Aggregates Inert?, Am. Ceram. Soc. Bull. 87, 3 (2008) 27-31.

[2] G. R. Rigby, R. F. Hutton,B. G. Hamilton, Reactions Occuring in Basic Brick, J. Am. Ceram. Soc. 46, 7 (1963) 332-342.

[3] L. Krietz, Refractories Handbook, Marcel Dekker Inc., EUA (2004) 259-285.

[4] C. Parr, L. Bin, B. Valdelievre, C. Wöhrmeyer, B. Touzo, The Advantages of Calcium Aluminate Cement Containing Castables for Steel Ladle Applications, Anais XXXII ALAFAR, Antigua, Guatemala (2004).

[5] M. A. L. Braulio, D. H. Milanez, E. Y. Sako, L. R. M. Bittencourt, V. C. Pandolfelli, The Effect of Calcium Aluminate Cement on the in situ Spinel Expansion, Proc., Dresden, Alemanha (2007) 540-543.

[6] M. A. L. Braulio, D. H. Milanez, E. Y. Sako, L. R. M. Bittencourt, V. C. Pandolfelli, Expansion Behavior of Cement-bonded Alumina-magnesia Refractory Castables, Am. Ceram. Soc. Bull. 86, 12 (2007) 9201-9206. 
[7] H. Sarpoolaky, K. G. Ahari, W. E. Lee, Influence of in situ Phase Formation on Microstructural Evolution and Properties of Castable Refractories, Ceram. Int. 28 (2002) 487-493.

[8] C. F. Chan, Y. C. Ko, Effect of CaO Content on the Hot Strength of Alumina-spinel Castables in the Temperature Range of $1000^{\circ} \mathrm{C}$ to $1500^{\circ} \mathrm{C}$, J. Am. Ceram. Soc. 81, 11 (1998) 2957-2960 .

[9] M. Fuhrer, A. Hey, W. E. Lee, Microstructural evolution in self-forming spinel/calcium aluminate-bonded castable refractories, J. Eur. Ceram. Soc. 18 (1998) 813-829.

[10] R. G. Pileggi, A. R. Studart, V. C. Pandolfelli, J. Gallo, How mixing affects the rheology of refratory castables, Part 1, Am. Ceram. Soc. Bull. 80, 6 (2001) 27-31.

[11] R. G. Pileggi, A. R. Studart, V. C. Pandolfelli, J. Gallo. How mixing affects the rheology of refratory castables, part 2. Am. Ceram. Soc. Bull. 80, 7 (2001) 38-42.

[12] Z. Guo, S. Palco, M. Rigaud, Reaction characteristics of Magnésia-spinel refractories with cement clinker. J. Appl. Ceram. Technol. 2, 4 (2005) 327-335.

(Rec.25/03/2008, Ac. 18/07/2008) 